Journal of Computer Science 7 (3): 431-433, 2011

ISSN 1549-3636

(C) 2011 Science Publications

\title{
Performance Analysis of IEEE 802.16d using Forward Error Correction
}

\author{
${ }^{1}$ Venkatesh Sekar, ${ }^{2}$ V. Palanisamy and ${ }^{3} \mathrm{~K}$. Baskaran \\ ${ }^{1}$ Department of Electronics and Communication Engineering, \\ Kumaraguru College of Technology, Coimbatore, Tamil Nadu, India \\ ${ }^{2}$ Department of Electronics and Communication Engineering, \\ Info Institute of Engineering, Coimbatore, Tamil Nadu, India \\ ${ }^{3}$ Department of Computer Science Engineering, \\ Government College of Technology, Coimbatore, Tamil Nadu, India
}

\begin{abstract}
Problem statement: Worldwide Interoperability for Microwave Access is the broadband wireless technology for terrestrial broadcast services in Metropolitan Area Networks. This BWA technology is based on Orthogonal Frequency Division Multiplex (OFDM) technology and considers the radio frequency range up to $2-11 \mathrm{GHz}$ and $10-66 \mathrm{GHz}$. The objective of this research work is the performance analysis of AWGN, SUI-1 and SUI-2 fading channels in IEEE802.16d using FEC to improving BER at different SNR under QPSK digital modulation technique. Approach: Channel coding part is composed of three steps randomization, Forward Error Correction (FEC) and interleaving. FEC is done in two phases through the outer Reed Solomon (RS) and inner Convolutional Code (CC). The implementation and analysis will be made on using MATLAB simulation. Results: In this analysis the Bit Error Rate under QPSK modulation technique over AWGN, SUI-1 and SUI-2 fading channel with encoder for a SNR value of $5 \mathrm{~dB}$ but in the case of without encoder is found SNR value of $9 \mathrm{~dB}, 10 \mathrm{~dB}$ and $8 \mathrm{~dB}$ respectively. Conclusion: It was concluded that all the three fading channel had same SNR with FEC .The optional Block Turbo Coding (BTC) can be implemented to enhance the performance of FEC.
\end{abstract}

Key words: OFDMA, Convolutional Code (CC), QPSK, Forward Error Correction (FEC), AWGN, Block Turbo Coding (BTC), Reed Solomon (RS), WiMAX, Line Of Sight (LOS), Signalto-Noise Ratio (SNR)

\section{INTRODUCTION}

The rapid growth of wireless internet causes a demand for high-speed access to the World Wide Web. To serve the demand for access to the internet "anywhere any time" and ensure quality of service, the IEEE 802.16 working group brought out a new broadband wireless access technology called WiMAX meaning Worldwide Interoperability for Microwave Access. It is the most recent solution for the provision of fixed broadband wireless services in a wide geographical scale and proved to be a real effective solution for the establishment of wireless metropolitan area networks. WiMAX will represent a serious competitor to third generation cellular systems as high speed mobile data applications will be achieved with the 802.16 specification. WiMAX is especially popular in wireless applications because of its resistance to forms of interference and degradation. In short,
WiMAX delivers a wireless signal much farther with less interference than competing technologies. The first version of the IEEE 802.16 standard operates in the 10$66 \mathrm{GHz}$ frequency band and requires Line Of Sight (LOS) towers. Later the standard extended its operation through different PHY specification to $2-11 \mathrm{GHz}$ frequency band enabling Non Line Of Sight (NLOS) connections, which require techniques that efficiently mitigate the impairment of fading and multipath (Koffman and Roman, 2002). Taking the advantage of OFDM technique the PHY is able to provide robust broadband service in hostile wireless channel. The channels are spaced very close together but avoid interference because neighboring channels are orthogonal to one another and thus have no overlap. Generally the Signal-to-Noise Ratio (SNR) requirements of an environment determine the modulation method to be used in the environment. QPSK is more tolerant of interference than either 16-

Corresponding Author: Venkatesh Sekar, Department of Electronics and Communication Engineering, Kumaraguru College of Technology, Coimbatore, Tamil Nadu, India 
QAM or 64-QAM (Hasan and Hossain, 2010). The implementation of RS encoder is derived from a systematic $\mathrm{RS}(\mathrm{N}=255, \mathrm{~K}=239, \mathrm{~T}=8)$ code using GF $\left(2^{8}\right)$.The encoder support shortened and punctured code to facilitate variable block sizes and variable error correction capability. The outer RS encoded block is fed to inner binary convolutional encoder has rate of $1 / 2$.

\section{MATERIALS AND METHODS}

The MATLAB simulation model consists of three main components namely transmitter, receiver and channel. Transmitter and receiver components consist of channel coding and modulation sub-components whereas channels are modeled as fading channels. This structure corresponds to the physical layer of the IEEE 802.16 2004 Wireless MAN OFDM air interface. WiMAX supports a number of modulation and Forward Error Correction (FEC) coding schemes and allows the scheme to be changed on a per user and per frame basis, based on channel conditions. Adaptive modulation and coding is an effective mechanism to maximize throughput in a time-varying channel. The adaptation algorithm typically calls for the use of the highest modulation and coding scheme that can be supported by the signal-to-noise and interference ratio at the receiver such that each user is provided with the highest possible data rate that can be supported in their respective links.

Channel coding part (Fig.1 and 2) is composed of three steps randomization, Forward Error Correction (FEC) and interleaving. FEC is done in two phases through the outer Reed Solomon (RS) and inner Convolutional Code (CC).Reed Solomon Encoder that encapsulates the data with coding blocks and these coding blocks are helpful in dealing with the burst errors. The block formatted (Reed Solomon encoded) data stream is passed through a convolutional interleaver. Here a code rate can be defined for convolutional codes as well. If there are $\mathrm{k}$ bits per second input to the convolutional encoder and the output is $\mathrm{n}$ bits per second, the code rate is $\mathrm{k} / \mathrm{n}$ (Venkatesh et al., 2010 and Bayan et al., 2010).

The following Table 1 shows the simulation parameters and its values used in MATLAB simulation model to analyze fading channels (AWGN,SUI-1 and SUI-2) using forward error correction scheme.

The radio architecture of a communication system plays very significant role in the modeling of a channel. The wireless channel is characterized by: Path loss, Multipath delay Spread, Fading characteristics, Doppler spread, co channel and adjacent channel interference.

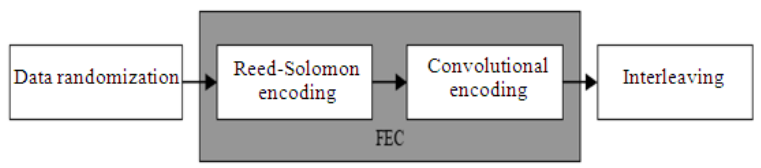

Fig. 1: Channel encoding

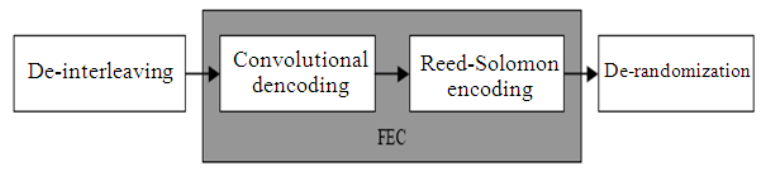

Fig. 2: Channel decoding

Table 1: Simulated Coding, Modulation Scheme and Noisy channels

\begin{tabular}{ll}
\hline Parameter & Value \\
\hline Nominal channel bandwidth & $2.5 \mathrm{MHz}$ \\
Cyclic prefix & $1 / 4$ \\
Number of used sub carriers & 200 \\
Modulation & QPSK \\
Channels & AWGN, \\
& SUI-1, SUI-2 \\
RS Code & $(255,239,8)$ \\
CC Code & $1 / 2$ \\
\hline
\end{tabular}

Table 2: Channel Model parameters

\begin{tabular}{ll}
\hline Parameter & Value \\
\hline P in dB & {$\left[\begin{array}{lll}0 & -15 & -20\end{array}\right]$} \\
K & {$\left[\begin{array}{lll}0 & -12 & -15\end{array}\right]$} \\
& {$\left[\begin{array}{lll}4 & 0 & 0\end{array}\right]$} \\
Tap delay & {$\left[\begin{array}{lll}2 & 0 & 0\end{array}\right]$} \\
& {$\left[\begin{array}{lll}0.0 & 0.4 & 0.9\end{array}\right]$} \\
Dop (Hz) & {$\left[\begin{array}{lll}0.0 & 0.4 & 1.1\end{array}\right]$} \\
& {$\left[\begin{array}{lll}0.4 & 0.3 & 0.5\end{array}\right]$} \\
& {$\left[\begin{array}{lll}0.2 & 0.15 & 0.25\end{array}\right]$} \\
Auto_corr & $0.7,0.5$ \\
Normalized factor & $-0.1771,-0.3930$ \\
of gain (dB) & \\
\hline
\end{tabular}

SUI channel models are an extension of the earlier study by ATaT Wireless and Erceg et al. (2001). This model can be used for simulations, design and development and testing of technologies suitable for fixed broadband wireless applications .The parameters for the model were selected based upon some statistical models. From these six channels we considered two channels for analysis (Erceg et al., 2001; Baum, 2001). The Table 2 below depicts the parametric view of the SUI-1 and SUI-2 channel.

\section{RESULTS}

For the purpose of this study, we have experimented with various kinds of simulations on MATLAB to understand Bit Error Rate (BER) versus the Signal to Noise Ratio (SNR) plots for QPSK modulation using 1/4 cyclic prefix. Figure from 3 to 5 shows the performance on AWGN, SUI-1 and SUI-2 channel models with and without encoder respectively. 
Simulation results in figure from 3 to 5 shows the advantage of considering a $1 / 2$ rated convolutinal coding and Reed-Solomon coding $(255,239,8)$ for the QPSK digital modulation scheme.

The following Table 3 shows the Bit Error Rate under QPSK modulation technique over AWGN, SUI-1 and SUI-2 fading channel with encoder for a Signal to Noise Ratio value of $5 \mathrm{~dB}$ but in the case of without encoder is found Signal to Noise Ratio value of $9 \mathrm{~dB}, 10 \mathrm{~dB}$ and $8 \mathrm{~dB}$ respectively.

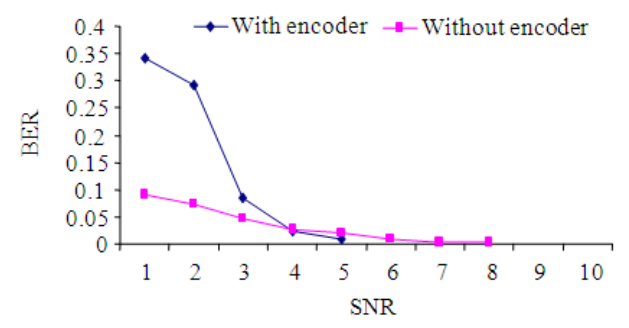

Fig. 3: Comparative study between with and without encoder using QPSK modulation under AWGN channel

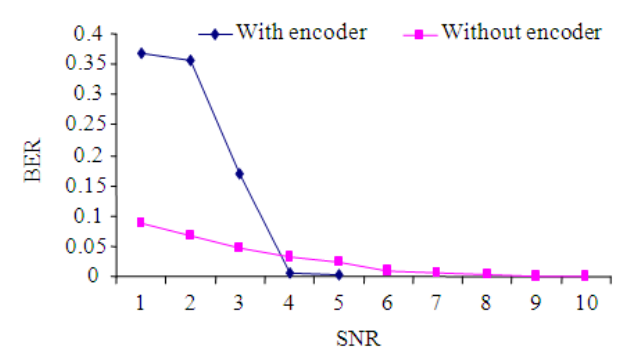

Fig. 4: Comparative study between with and without encoder using QPSK modulation under SUI-1 channel

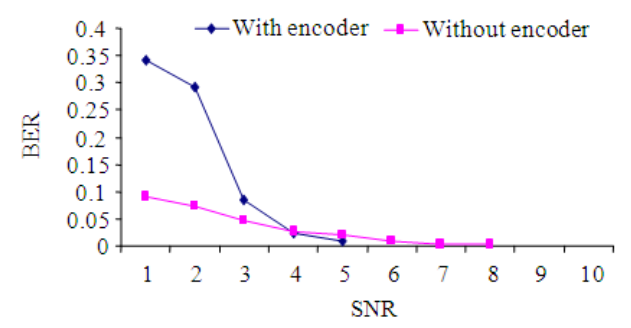

Fig. 5: Comparative study between with and without encoder using QPSK modulation under SUI-2 channel

Table 3: SNR in $\mathrm{dB}$ using QPSK modulation with and without encoder

\begin{tabular}{llll}
\hline & SNR $(\mathrm{dB})$ & & \\
\cline { 2 - 4 } & AWGN & SUI-1 & SUI-2 \\
\hline With & 5 & 5 & 5 \\
Encoder & 5 & 10 & 8 \\
Without & 9 & & \\
\hline
\end{tabular}

\section{DISCUSSION}

Broadband Wireless Access (BWA) has emerged as a promising solution for last mile access technology to provide high speed internet in the residential as well as small and medium sized enterprise sectors. In this study, we have analyzed the performance improvement due to convolutinal coding and Reed-Solomon coding on QPSK modulation and coding profiles has been observed on AWGN, SUI-1 and SUI-2 channel model. The same method can be used to analyze the BER performance of other SUI channels also.

\section{CONCLUSION}

The comparative analysis of the Bit Error Rate under QPSK modulation technique over AWGN, SUI-1 and SUI-2 fading channel with Forward Error Correction for a Signal to Noise Ratio value of $5 \mathrm{~dB}$ but in the case of without encoder is found SNR value of $9 \mathrm{~dB}, 10 \mathrm{~dB}$ and $8 \mathrm{~dB}$ respectively. All the three fading channels had same Signal to Noise Ratio of $5 \mathrm{~dB}$ with FEC .The optional Block Turbo Coding can be implemented to enhance the performance of Forward Error Correction.

\section{REFERENCES}

Baum, D.S., 2001. Simulating the SUI Channel model. IEEE 802.16.3c-01/53. http://wirelessman.org/tg3/contrib/802163c01_53.pdf

Bayan, A.F., T.C. Wan and S. Ramadass, 2010. Delay analysis and system capacity control for mobile WiMAX relay networks. J. Comput. Sci., 6: 1137-1143. DOI: $10.3844 /$ jcssp.2010.1137.1143

Erceg, V., K.V.S. Hari, M.S. Smith, D.S. Baum et al, 2001. "Channel Model for Fixed Wireless Applications. IEEE 802.16.3 Task Group Contributions.

http://www.ieee802.org/16/tga/docs/80216a03_01.pdf

Hasan, M.Z. and M.R. Hossain, 2010. Performance evaluation of a concatenated FEC scheme based WiMAX system with application to the digital image transmission over white guassian noise and fading channels. Asian J. Inform. Technol., 9: 62-66.

Koffman, I. and V. Roman, 2002. Broadband wireless access solutions based on OFDM access in IEEE 802.16. IEEE Communi. Magazine, 40: 96-103. DOI: $10.1109 / 35.995857$

Venkatesh, S., V. Palanasamy and K. Baskaran, 2010. Comparative study of SUI channels in IEEE802.16d using different cyclic prefix. Eur. J. Sci. Res., 48: 305-314. 